\title{
Escalation vs. Non-Escalation of Fan Violence in Football? Narratives from Ultra Fans, Police Officers and Security Employees
}

\author{
Alain Brechbühl, Annemarie Schumacher Dimech, Olivier Schmid, \& Roland Seiler \\ Institute of Sport Science, University of Bern, Bern, Switzerland
}

Corresponding author:

Alain Brechbühl

Email: alain.brechbuehl@ispw.unibe.ch

Tel: +41316315183

Fax: +41316315169 


\begin{abstract}
Despite a large body of literature about fan violence issues, research investigating perceptions and dynamics in potentially violent situations, called critical incidents (CIs), is missing (Schumacher Dimech, Brechbühl \& Seiler 2016). This qualitative study examined the perceptions and dynamics of CIs involving ultra football fans. Fifty-nine semi-structured interviews with individuals (fans, police officers or security employees) involved in eight CIs were conducted and analysed using Interpretative Phenomenological Analysis (Smith, Flowers \& Larkin 2009). The assessment of legitimacy of an out-group's actions strongly influenced the perception of a CI, which is in line with the Elaborated Social Identity Model (Reicher 1996b). Informative intergroup communication about the own intentions seemed to increase perception of legitimacy, while arrests of fans due to pyrotechnics were perceived by fans as illegitimate. The local fan culture, e.g. the fans' use of pyrotechnics for their support, is relevant for the understanding of this assessment of legitimacy.
\end{abstract}

Keywords: fan violence; ultras; critical incidents; perceptions; group dynamics

\title{
Introduction
}

European football draws countless fans to the stadium on a weekly basis. Besides the events on the field, recurring violent incidents involving football fans attract public and media attention and also raise concerns at a political level (Havelund et al. 2013). In this context, "physical violence" includes two essential elements: the actor's intention to behave violently and the damage to a person or object (Nolting 2005, 16). Substantial amounts of money and resources are invested yearly in the prevention and management of fan violence (e.g. Mensch and Maurer 2014). Police estimates report a population of approximately 600 to 700 violence-seeking and 1800 to 2000 potentially violent 
individuals attending football matches in Switzerland (Bundesamt für Polizei fedpol 2015). According to the Federal Office of Police (Bundesamt für Polizei fedpol 2014), ultra fans are responsible for most escalations in the Swiss football national championship. There is no standard definition of ultras ${ }^{1}$ due to cultural and local differences. Most authors in this field (e.g. Pilz and Wölki-Schumacher 2010) distinguish between moderate and more radical ultras. Radical ultras admit to violent behaviour and perceive the police as an enemy while moderate ultras are more restrained. In contrast to violence-seeking fans, such as hooligans who explicitly seek experiences of violence, ultras resort to violence for self-defence purposes or to achieve a certain goal (e.g. to "mark out their territory"; Pilz and Wölki-Schumacher 2010, 18). All ultras nevertheless share several characteristics. They assign a high level of importance to football and their club and attend all of their team's matches. They contribute to the atmosphere in the stadium by unique means of choreographies, chants, flags, banners, and pyrotechnics. Ultras also militate against the commercialisation of football and consider their presence as essential for their team's success (Havelund, Joern, and Rasmussen 2012; Pilz 2005).

Recent research examining causes of fan violence focused on the patterns and dynamics of interactions between involved groups (security, police and fans) through the lens of the Elaborated Social Identity Model (ESIM; Reicher 1996b; Stott, et al. 2007; Stott, Hoggett, and Pearson 2012) or the Aggravation Mitigation Model (AMM; Granström et al. 2009). The ESIM highlights the importance of public order policing for the development of football fan violence. According to the ESIM, the fans' perception

\footnotetext{
${ }^{1}$ For example, for Italy see Dal Lago and De Biasi 1994; for Germany see Pilz 2005; for Denmark see Havelund, Joern, and Rasmussen, 2012. An extensive comparison of different facets of the ultra culture(s) is available in Pilz and Wölki-Schumacher 2010.
} 
of the police actions' legitimacy is crucial: The intentions and actions of a group are under constant interpretation and re-assessment by the out-group. An action by the police interpreted as offensive and illegitimate may lead to a change in the fans' social identity and unite different groups of fans to form an entity (Stott et al. 2007). Social identity refers to the perceived membership to a social group, which contributes to an individual's self-concept (Reicher 1996a) and is defined as a "model of one's position in a set of social relations" (Drury and Reicher 2000, 581), which also includes appropriate actions for this position (e.g. to support the favourite team as a fan). A change of a fan's social identity could mean that violence is suddenly considered as a legitimate reaction (Stott, Hutchison, and Drury 2001). The united fan crowd could "feel both motivated and empowered to challenge these outside forces" (e.g. the police; Reicher 1996b, 130).

Several studies used the ESIM in the context of football related violence. Low profile policing (few visible police officers with riot gear) was found to be beneficial for the prevention of fan violence and contributed to a festive atmosphere and to selfregulation tendencies among fans (Stott et al. 2007; Stott et al. 2008). In another study, Stott, Hoggett, and Pearson (2012) using an ethnographic approach concluded also that the switch in policing from "deterrence" to "dialogue" improved the fans' perception of legitimacy and their relationship with the police. The violence-seeking fans' influence on the other fans decreased and fans even reported organised confrontations between hooligans to the police.

The Aggravation Mitigation Model (AMM (Granström et al. 2009) complements to the ESIM (Reicher, 1996b) by categorising the intra- and intergroup interaction processes in crowd situations into three interacting types: (1) Categorization refers to 
the assignment of a negative stereotype to the out-group (e.g. describing them as a potential threat) while the in-group is humanised (aggravation), or the differentiation between the out-group members (to acknowledge that the crowd consists of different groups with different intentions; mitigation). (2) Organisation is characterized by creating chaos (e.g. one group acting in an unexpected manner; aggravation) or creating order (e.g. providing information to the out-group; mitigation). (3) Treatment refers to (un)intentional provocation or "letting oneself be provoked" (Rosander and Guvå 2012, 57). Mitigation can be defined as acting peacefully by renouncing from use of weapons or symbols of power. The occurrence of all three aggravating processes is required for an escalation (Hylander and Granström 2010). Studies based on the AMM also underline the advantages of low profile policing and the establishment of dialogue with the fans to prevent violent escalations (Hylander and Granström 2010). The negative effect of stereotyping is also mentioned by Havelund, Joern, and Rasmussen (2014) and they recommend that less fan-experienced uniformed personnel (in contrast to more experienced police intelligence units) should gain a deeper understanding of the different fan cultures to avoid negative stereotyping. Considering the importance of group perceptions stated in the ESIM (Reicher 1996b) and the AMM (Granström et al. 2009), further investigations of perceptions of potentially violent situations are essential for effective prevention of football related fan violence. Since both models highlight the interdependency of the group actions, it can be argued that potentially violent situations, referred to here as "critical incidents" (CIs) (Schumacher Dimech, Brechbühl \& Seiler 2016), follow an unpredictable dynamic. No study so far has examined the origin and development of CIs around football matches from different perspectives (e.g. fans, police officers, security employees). Additionally, most fan violence studies have been 
conducted at matches involving national teams (e.g. Rosander and Guvå 2012; Stott, Hutchison, and Drury 2001; Stott et al. 2007), whereas fan violence typically occurs at domestic football matches of the highest national championship leagues (e.g. Germany or Switzerland). Stott, Hoggett, and Pearson's study (2012) is one of the few investigations including domestic football fans, however it did not exclusively focus on ultras. Considering ultras' unique supportive culture and use of pyrotechnics, the origins and development of CIs involving this group might be different than with other fans. The current study addresses these limitations through an analysis of CIs involving ultra football fans in Switzerland.

The purpose of the current study is to examine the perceived causes and dynamics of violent escalations following CIs in the Swiss premier football league matches in Switzerland. A qualitative design was used to gain deeper understanding of how perceived experiences and interpretations of individuals involved in CIs, including football fans, security employees (SEs) and police officers (POs), influence the escalation or non-escalation of inter-group fan violence. Due to the ultras' prominence in Switzerland's fan scene, this study focusses on CIs involving ultras at domestic matches. The following research questions are addressed. (1) How do fans, POs and SEs perceive the characteristics of a CI and its development? (2) What differentiates escalating from non-escalating CIs? 


\section{Method}

\section{Data collection}

After obtaining ethical approval by the Faculty's Ethics Committee, the Swiss fan social workers $^{2}$ (FSWs) of two of the largest Swiss premier league clubs agreed to participate in this study during the 2012/2013 season. Their acceptance was essential to obtain access to the fans. Both football clubs have a dominant ultra fan scene and at least one capo $^{3}$. The first author held meetings with the two clubs' FSWs, the fan workers ${ }^{4}$ (FWs) and security staff to build a rapport and inform them about the purpose of the study. To build rapport with the fans, the first author attended three matches (per team) in the fan zone, and discussed the aim of the study and issues of anonymity with the most important fans. The first author also met the local and national competence centres of the police. All stadium officers in Switzerland were informed in writing about the purpose of the study and their support was granted in the event of a critical incident in their stadium.

Schumacher Dimech, Brechbühl, and Seiler (2016) introduced the term "critical incident" (CI) to describe a setting in or around the football stadium, in which violence between two or more groups could occur, and defined observable criteria to identify them. A CI can either lead to violence escalation or non-escalation, where an initially tense situation simmers down without any violence. In this study, the term escalation refers to violent acts involving two groups, for example fans throwing objects at the police who react by firing rubber bullets. A total of eight CIs at away matches were

\footnotetext{
${ }^{2}$ Fan social worker: socio-professional fan worker paid by the "Trägerverein" [support association] and the particular canton and city (Projektgruppe „Rahmenkonzept Fanarbeit in der Schweiz“ 2010).

${ }^{3}$ Common name for the group leader of ultras, who leads choruses and chants during matches.

${ }^{4}$ Fan worker: club-related fan worker working for and paid by the football club (Projektgruppe „Rahmenkonzept Fanarbeit in der Schweiz“ 2010)
} 
identified onsite by the first author, FSWs and police spotters ${ }^{5}$ between October 2012 and June 2013 (each team played 15 away-matches in this period). An incident was considered to be critical if an intervention by police officers (POs), security employees (SEs) or fans aimed at influencing or controlling the other group's actions occurred. Five CIs were identified at away matches of football club 1 and three at away matches of football club 2 .

\section{Participants}

Within three weeks following the identification of a CI, individuals directly or indirectly involved were contacted and interviewed. Contact with participants was established through the FSWs (fans;), stadium officers (SEs) and local competence centres (POs). In every case, the perspectives of the fans, FSWs, and POs or SEs were represented. All participants were informed about the purpose of the study and signed an informed consent form outlining their rights as participants.

\section{Interviews}

A narrative approach was used to interview the participants. To stimulate recall processes and elicit narratives, a brief description of the respective CI was presented to the participants. Participants were asked to tell the story of the case from their own perspective. After participants had finished their stories, follow up questions were asked about the course of action, the perception of the in- and out-group(s), the participant's own actions and their attribution of what may have caused the escalation or nonescalation of the CI. The interviews lasted between 15 and 105 min, were carried out in

\footnotetext{
${ }^{5}$ Police spotters are experts on fan groups of their local football club. They deliver information to the director of operations of the police. Spotters are also responsible for the surveillance of known offenders and usually work in plain clothes.
} 
Swiss German or French by the first author and were audio recorded. Fifty-nine interviews were conducted with 35 different persons, including nine fans, two FWs, three FSWs, 13 POs and eight SEs.

\section{Data analysis}

The interviews were transcribed verbatim. Interview transcripts were kept anonymous and were sent to each participant for feedback. Besides minor edits, no changes or corrections were requested. For the present paper, quotations were translated by an English expert. Data analysis was conducted in two separate steps.

\section{Step 1: case descriptions}

Case description summaries were compiled for each interview and the case's story line was separated in different scenes by the first and second author (e.g. "the search for the fans at the stadium"). A case includes all events occurring on the day of the match (the journey to the match, the match itself, and the fans' way back home). The CIs were further categorised by the first and second author into three different developmental phases (Figure 1): "the onset of the CI", "the peak of the CI" and "the pacification of the CI" or "the escalation of the CI"

[Figure 1 near here]

The CI's onset occurred when one group tried to influence the actions of the outgroup. The peak marked maximum level of tension without physical violence occurring between the groups. In CIs ending in an escalation, the outbreak of violence took place 
after the peak of the CI. In CIs without escalation, tension decreased and resulted in a pacification.

The case descriptions included in Table 1 and 2 are based oncommonalities in the participants' narratives. Some incongruences in the participants' case descriptions were observed regarding the actual distance between the groups (Case 2), group size (Cases 1, 3 and 5), the perceived aggression of a group (Cases 2, 4, 5, 7, 8) or the communication between the groups (Case 4).

[Table 1 near here]

[Table 2 near here]

\section{Step 2: Interpretative Phenomenological Analysis}

Interviews were analysed by the first author using Interpretative Phenomenological Analysis (IPA; Smith, Flowers, and Larkin 2009). This phenomenological approach uses a double hermeneutic, in which the researcher interprets the way participants make sense of their experiences. An inductive analysis approach was conducted regarding the participants' perception of what can lead to aggression, of their emotional states, their situation appraisal processes, their chosen reactions, and consequences. Themes were built and integrated into the combined scene descriptions from step 1. Upon analysis of each case, comparisons between the eight different cases were conducted to identify recurring themes leading to escalation and non-escalation.

To increase trustworthiness and consistency of the interpretations, different research team members were involved in the coding and interpreting process of eight interviews. 
In instances of diverging interpretations, the differences were discussed until meaning units and interpretations of all members converged.

\section{Analysis}

First, themes related to the different groups' perceptions in critical incidents (CIs) are presented. The themes relate to the personal space, the out-group behaviour, symbols, legitimacy, and fan culture. Second, differences between critical incidents with escalation and without escalation are brought forward, including the discussion of dynamics.

\section{The perceptions of the critical incidents}

Group-specific perception of the fan group's personal space

Fans expressed a clear sense of their group's personal space. They labelled physical distance (space) to the out-group as sufficient or insufficient. In Case 2 (no escalation), the interviewed fans complained about the insufficient distance maintained by two police spotters escorting the fanwalk. A verbal argument ensued between fans and the spotters, whereupon the spotters had to retreat due to their inferiority in numbers. Fans described the spotters' behaviour as "walking inside the fanwalk", whereas the spotters stressed that they "...were [...] at a distance of about 100 meters and followed it the fanwalk]" (Case 2, PO1). The spotters' approach at the CI's onset in Case 2 was perceived as an intrusion into the fans' area (the fanwalk), eliciting anger among the fans while radical fans prepared for a possible attack. Several fans described the spotters' approach as an intentional provocation. "...when the police get too close to us, it is a provocation. Because both sides know exactly that those on the other side are not 
approachable" (Case 2, Fan5). Fans' past experiences seem to be a key element in the assessment of the spotters' actions. The interviewed fans from Case 2 highlighted their conflictual relationship with the local police spotters due to past negative experiences such as arrests of fans. Therefore, it appears that past experiences influence future interactions between the groups.

Perception of the out-group's behaviourBased on these past experiences, some fans and police officers (POs) or security employees (SEs) reported their negative perceptions of the out-group. For example, fans mentioned their general distrust in the POs and SEs and interpreted their behaviour as aggressive and provocative. In Case 2, fans talked about their aversion to police spotters from their home town. "I know however that they [POs] follow other aims than we pursue, respectively, completely different ones. They want to [...] destroy our fan culture and I cannot accept that.” (Case 2, Fan5). Another fan reflected the relation to the police spotters as follows: “...one does not like them much. Simply because they are police spies and try to identify people and even lead to people being banned." (Case 2, Fan2). Some fans seem to hold a negative stereotype ${ }^{6}$ of their respective out-group by describing them as homogeneous and possessing negative characteristics. Most stereotypes were related to the local police or a specific security company. Three fans described POs and SEs as their enemies: "Well, we fundamentally have a rather hostile attitude towards state and security institutions [...]. Those are red flags." (Case 7, Fan7). Other fans and fan social workers (FSWs) confirmed that this view is widely shared by ultra fans. All fans complained about excessive court sentences and about being prosecuted for minor offences (e.g. verbal insults).

\footnotetext{
${ }^{6}$ Stereotyping means building stereotypes. Definition of a stereotype: "Associations and beliefs about the characteristics and attributes of a group and its members that shape how people think about and respond to the group" (Dovidio, Hewstone, Glick, and Esses 2010, p. 8).
} 
Likewise, several POs and SEs expressed a negative perception of the fans' behaviour by describing them as rude and potentially violence-seeking.

They get out of the trains like animals. $[\ldots]$ Now, $[\ldots]$ the first man in uniform they see, be it Securitas [...] or a police officer or a fireman, they will just (gesticulates a blow) give him hell. (Case 4, SE2)36

The tendency to stereotype was not the same for all POs: PO8 (police spotter) highlighted the fans' interest in their club's matches and only described a minority of fans as being violence-seeking. In contrast, PO11 (riot-gear unit) described the majority of the fans as violence-seeking. POs wearing riot-gear uniforms generally expressed more negative perceptions of the fans than police spotters.

Past experiences seemed to be essential for the negative perceptions of the outgroup.

An earlier incident was followed by various house searches, in which fans were dragged out of bed at 6 a.m. or were called from their place of work [...] and this was ultimately possible through the police spotters' investigations. One has a tense relationship with them because of this (Case 2, Fan1).

Negative experiences with the out-group tended to spread quickly across the ingroup and can become part of the in-group's identity: “...or the new security company in the city, where we had trouble last time. [...] They will also be a red rag in the future" (Case 4, Fan7). Negative stereotyping can be considered as essential for the assessment of the out-group's actions during the CI. In this case, the out-group's intentions tended to be perceived and interpreted as aggressive, which seemed to aggravate the CI. 


\section{Group-specific perception of provocative symbols}

All groups discussed the physical appearance of out-group's members, which was interpreted as aggressive. Six SEs and four POs reported that masked fans (wearing balaclavas, scarves or hoods) were symbols of aggression and acknowledged expectations of delinquent or hostile behaviour from these fans. "They masked themselves, put on their gloves and balaclavas or scarves. That is a sign of violence for me." (Case 2, PO4). These interpretations seemed to have an aggravating effect on the development of the CI. Nevertheless, one fan explained that the masking is often necessary for mediating individuals to avoid erroneous criminal prosecutions or to protect oneself from riot control measures (e.g. pepper spray and rubber bullets).

\footnotetext{
You are actually unmasked in the midst of this pepper spray theatre and you try to hold back people and explain to them that this is of no use etc. You place yourself at risk of cropping up in a video, perhaps as a ringleader. (Case 6, Fan6)
}

Fans seemed to be aware of the effect of masking themselves. In Case 8, a group of unmasked fans aided several masked fans by just standing in front of them (thus acting as a cover) in retrieving previously hidden pyrotechnical materials inside the stadium. The interviewed SEs perceived the unmasked fan group as non-aggressive and decided not to intervene.

There was no potential for violence in the front, it was more in the middle [of the fan group] [...]. And SE9 acted just perfectly by saying: 'there's no confrontation, so I don't see any reason to put on our helmets or to advance with our batons' (Case 8, SE8).

Conversely, the fans assessed the masking (used by some SEs in Case 5) and the SEs' or POs' riot-gear uniform (body armour, helmet, shield, and baton) as provocative. 
"The appearance of this outfit shows that they are ready. [...] One wears it so as not to be attacked by the fans. So one does expect being attacked. One actually provokes that." (Case 4, Fan3). In Cases 4, 5, and 8, the appearance of POs or SEs with riot gear triggered anger on the fans' side and seemed to aggravate the CI.

Similar to the fans, SE4 expressed being aware of the fans' interpretation, but highlighted the protective purpose of the riot-gear: "They take it as an aggression. We take it as a safety measure. We can't [...] face them without any protection" (Case 5, SE4). He further explained that they sometimes use the intimidating effect of the uniforms to demonstrate determination to act.

\section{Fans' assessment of legitimacy}

The fans' critical assessment of the legitimacy of the out-group's behaviour was observed with regard to all eight CIs and seemed to be determinant for the onset of a CI. Security employees or POs acting aggressively against fans was considered illegitimate. “...I had no understanding for the actions, for the use of pepper, and [...] I felt an excitement that flowed into aggression. I reached this point relatively quickly, where I ascribed the security staff with acting deliberately." (Case 4, FSW2). The perception of illegitimacy triggered feelings of anger. In some cases, the fans acknowledged the POs' behaviour as legitimate. In Case 4, POs reportedly intervened with batons in a brawl between two rival fan groups and retreated as soon as the fans were separated: "In my view, this police intervention was completely professional. They had to be there, they resolved it and it was good." (Case 4, Fan4). In contrast, measures like disproportionate 
searches (taking off shoes or searching the genital area) or the detention ${ }^{7}$ of fans, were generally perceived as illegitimate.

Most POs and SEs did not assess the fans' actions as being legitimate or illegitimate, although five of them expressed their incomprehension when observing violent acts by fans. The majority of the POs endorsed their professional role by emphasizing their need to focus during the CI: "When the escalation starts, I think, nobody is scared any more. Then it is just about fulfilling your task, I must do this and this [...], I must protect my partner, I must protect myself..." (Case 7, PO12). The taskfocused and emotional control demonstrated by the POs and SEs may be attributed to extensive professional experience. For example, PO10 placidly explained the attack of the fans and his interventions without any emotions or feelings. "For my part, I didn’t have any feelings, no fear or no animosity against those people [the fans]. It was really like going there and separating those groups" (Case 4, PO10). He appeared to be used to dealing with violence: "Those who kicked would get hit with the baton and so on. So yeah, that's it, hit with it... [...] About 2-3 blows were given using batons" (Case 4, PO10). However, PO1, PO4 (both in Case 2), PO11 (Case 4), and PO13 (Case 6) mentioned uneasiness when confronted with fans behaving aggressively: "A lot of respect, and I think especially with such a crowd involved, this can merge into fear later on" (Case 6, Pol13)“.

\section{Fan culture as a relevant factor}

The fans' general condemnation of illegitimate measures raised questions about their culture, which influences their judgements. In this section, the fans' statements about

\footnotetext{
${ }^{7}$ Detentions are conducted in several stadiums all around Switzerland after the end of a match to ensure a proper separation of the opposing fan groups. The fans are obliged to wait inside the stadium until the home fans have largely left their sector.
} 
their fan culture are discussed to get a better impression about their shared beliefs and opinions. All fans emphasised their attachment to their respective football club and their interest for the actual matches. Five fans reported being held in low esteem in Swiss society. "This is just the way football fans are viewed. You could throw a beer bottle and get a two-year stadium ban. But when a fan is [...] beaten up at the train station, nothing happens." (Case 1, FSW1). The interviewed fans expressed scepticism towards outsiders and highlighted the media attention on fan violence and the strict penalties for delinquency at football matches in this context.

While some of the interviewed fans expressed a moderate attitude towards the use of violence, and even acted as mediators, three fans were more radical and did not reject using violence. Despite these differences, most fans seemed to display great solidarity overall. FSW3 identified the support of the team as the fan group's common denominator The participants reported a hierarchical structure within their fan groups based on seniority. The "capos" were reportedly the most experienced and influential fans on their fan group's behaviour (e.g. as violence seekers or mediators).

All interviewed fans considered pyrotechnic equipment as essential to support their team, despite its illegal use. "...they [the pyrotechnics] really belong to our fan culture. It is not just an empty word. And yes, the police perceive this as an act of violence. [...] It is just really hard to understand.” (Case 2, Fan5). Possession and use of pyrotechnics are severely penalized and searches are enforced at the stadium entrance. Due to the severe penalties, fans seemed forced to increase their efforts to smuggle pyrotechnics into the stadium (e.g. Case 8) or to attempt to free a group member after a pyrotechnics related arrest: 
One tries everything to get him out in some way, and by whatever means [...] Of course it is also about the fact that we are stronger than the others, that we can free him. That is $[\ldots]$ a test of strength, $[\ldots]$ a power game between us. (Case 6, Fan5).

Fan7 considered the smuggling of pyrotechnics to be part of a power game with SEs: "This is just a guess, but it is a bitter defeat for them [the SEs] when we light about 100 flares in there. [...] Then we spray their stadium and they are simply powerless." (Case 4, Fan7). Fans considered such acts as an affirmation of superiority over the outgroup.

\section{Differences between escalations and non-escalations}

This part of the analysis discusses differences that emerged in the comparison of the interactions in escalating and non-escalating CIs. Table 3 illustrates occurrences that, according to the participants, played a crucial role in the development of the CIs.

[Table 3 near here]

The appeasing occurrences seemed to raise the perception of legitimacy of the out-group's actions. An example is informative communication between the involved groups, which had been reported by fans, POs, and SEs. If successful intergroup communication occurred during the CI, the CI was more likely to end without an escalation (e.g. Case 3 with successful communication between fans and POs, in contrast to Case 4 with unsuccessful communication between fans and SEs). The communication had to contain information about the intentions of the out-group in the incident itself. The fans valued knowing what to expect from the out-group. In Case 3, Fan2 appreciated that the coordinating PO was wearing an orange vest, so all fans were 
able to recognize him. The PO constantly informed fans about the negotiations that were going on in the background (between the police, fan workers, and the football club in order to permit the fans to talk to the team), which was praised by Fan5. In Case 4, the information about the fans' detention after the game was not forwarded to the fans and FSWs. The fans felt unfairly treated by the SEs. Informative communication tended to raise the perception of legitimacy of the out-group's behaviour, as stated by PO3 in Case 3: "I must say that the fans behaved in an exemplary manner, as they sought a dialogue and explained that they wanted to talk to the team now" (Case 3, PO3). It could also be interpreted that informative communication gave fans more time to assess their own reactions in a CI. For example, Fan7 admitted to impulsive violent behaviour:

I experienced a situation where they [the police] tried to get hold of a friend of ours for $[\ldots]$ no reason at all. That's the one with the fence flag. Then it goes wham! I ran unmasked towards the police and kicked at their shields, pulled my friend out. On looking back, one realises: you damned idiot. I mean, one could easily get banned from the stadium for something like that. (Case 7, Fan7)

Based on the participants' narratives, it could be argued that in cases where the POs or SEs were successful in establishing the fans' perception of legitimacy, successful mediation by FSWs, fan workers (FWs) or fans was most likely to occur. Conversely, when the fans perceived the POs' or SEs' actions as illegitimate, they reported stopping their efforts to control their own fans.

For those [reasons], where the fans' reaction is not legitimate, I try to intervene and say that it is of no use. [...] But [...] when I cannot understand either and when I can identify [...] with those who want to get him [the arrested fan] out [...], I do not intervene. (Case 6, Fan5) 
If fans perceived themselves to be negatively stereotyped (i.e. treated as a homogenous and aggressive group), this was considered as illegitimate. Especially the indiscriminate use of riot control measures seemed to prevent peaceful fans and FSWs from keeping their composure. Fans, POs, and SEs seemed to assess the out-group's actions as more legitimate when the out-group refrained from displaying provocative symbols (e.g. riot gear or balaclavas). In Case 8, the SEs perceived a group of fans as peaceful who deliberately refrained from masking themselves to form a buffer in front of the more radical fans. By not wearing their helmets, the SEs also tried to reduce the debatably provocative appearance and the CI ended without an escalation.

Unsurprisingly, the retreat of an involved group seemed to dissolve a CI. According to the CIs descriptions, such instances occurred after intrusions into the fans' personal space. The retreating group perceived themselves to be in an inferior position compared to the out-group. In Cases 2 and 8, the POs and SEs retreated due to their inferiority. In Case 5, the participants reported a face-off between a group of fans and SEs in the searching area. The fans assessed the confrontation as impossible to win and retreated into the stadium. According to Fan5, this "defeat" caused aggression but did not lead to an escalation between the groups: "We got in. 3, 4 [fans] kicked the fence and all that. They just let off some steam" (Case 5, Fan5).

Several aggravating occurrences (see Table 3) appeared to contribute to the perception of illegitimacy, including the arrest of a fan due to pyrotechnics or a rival group physically attacking the fans. According to all the interviews in Case 6, a fan's arrest for possession of pyrotechnical materials usually leads to an escalation. "I know what consequences he has to face. He did something that is prohibited by the law, but it 
is just part of things for us and I fully support this" (Case 6, Fan6). Fan5 even mentioned that the arrest of a fan in general (not only due to pyrotechnics) leads to an escalation: "Nobody prevents it [the fans' violence] and [...] so there it always escalates. Not always to the same extent, clearly. But it always leads to an escalation in this sense." (Case 6, Fan5). The fans considered pyrotechnics as essential element of their culture. Accordingly, the fans seem to perceive an arrest of a fan due to pyrotechnics as an illegitimate attack. A violent reaction by the in-group was perceived as legitimate self-defence: "Getting someone out [a fan under arrest]... I actually always find that legitimate. These are also the incidents where the situation escalates." (Case 6, Fan5). Several participants (five fans, two FSWs, two FWs, one SE, and three POs) mentioned that attacks from other groups would always be retaliated by the fans.

Provocations from rival fans were another aggravating occurrence that appeared in the interviews. In Case 7, Fan9 and Fan7 stated that the attempt to conduct the fanwalk was triggered by previous provocations from the rival fans. Fan9, who watched the match in Case 7 at a restaurant close to the stadium due to a stadium ban, explained:

[...] those who seek this are wild about it [a brawl] and want to go for it. It was promised and then nothing happens. There is a relative aggression due to that, it starts to build up. They [the rival fans] act stupidly on the way to the stadium. Then one comes over to see what kind of people are here and states his intentions [of a brawl]. Then the phone calls start and nothing happens. What do they want?! (Case 7, Fan9)

A fight was announced by the rival fans. Once advertised, some fans seemed to actively seek conflict. Fans and FSWs explained that in ultra culture, backing out of a conflict is cowardly and illegitimate, even more so when the provoking group backs out. In Case 7, Fan7 and Fan9 reported feeling frustrated in the absence of a fight against 
rival fans after the match after provocations from the rival rival fans, even though the former had stated no interest in an escalation before the match. Fans, FWs, FSWs, and POs stated that conflicts with rival fan groups are an essential part of the ultra fan culture and that some fans try to present themselves as a powerful group.

In a way we were frustrated. [...] as our club is a club that is always made fun of and not taken seriously, especially by outsiders. And then something like this [provocations from rival fans] comes along and nothing happens. (Case 7, Fan9)

Arch-rivalry between opposing fans seemed to further increase the likelihood of violent reactions. In Case 5, no escalation was reported despite the mentioned archrivalry with the opponent fan group. However, FW1 referred to a violent clash prior to the match between rival fans. FSW2 also mentioned an appeasing effect of pre-match fan conflicts.

\footnotetext{
"It is not a serious solution, but it is something that could defuse the situation, when such a meeting [between rival violence-seeking fans] is possible before a match. This way, the day of the match is calmer." (Case 7, FSW2).
}

The presence of violence-seeking fans was believed to be another essential occurrence related to the outbreak of an escalation (e.g. in Case 4 or 7). Their presence in Case 7 led to pessimistic expectations of several fans and the FSW prior to the match. The participants further explained that some radical fans sought conflict with rival fans and were able to influence the route of the whole fan group at the onset of the CI in Case 7.

\footnotetext{
...there are people from this scene that have quite a lot to say and look up to a couple of youngsters from the hooligans $[\ldots]$ they join in without thinking. In addition, mostly it is associated with an existing image of the enemy. So against arch-rivals, where $[\ldots]$ the normal ultra would gladly score a notch or punch a rival
} 
fan in the face, but he $[\ldots]$ has little courage to act at this moment $[\ldots]$ and then is glad, when a couple of hooligans take over, who have no fear. They then walk with them in the second or third row. (Case 7, FSW2)

Mutual influence tends to exist between the violence-seeking fans (hooligans) and the ultra fans and they seemed most likely to join forces against an arch-rival. Handling conflicts between rival fans appeared to be complex for POs. According to the narratives about Case 7, POs tried to seek dialogue with the fans during the CI. Although the interviewed fans perceived the actions of the police as legitimate, other radical fans reportedly sought hostile actions with opponent fans and tried to initiate a fanwalk. This conflict only affected a smaller group of fans, while most fans soon retreated to the buses (after realising that their attempt to conduct a fanwalk was ineffective).

\section{Discussion}

The purpose of this investigation on fan violence in the Swiss premier league was to (1) explore perceived experiences of nine fans, two fan workers (FWs), three fan social workers (FSWs), 13 police officers (POs), and eight security employees (SEs) who experienced a critical incident (CI) involving ultra fans and to (2) analyse what differentiates escalating and non-escalating CIs. The data suggests that perceptions are often similar among members of the same group (e.g. fans or POs). The types of interactions between the involved groups and the resulting dynamics can be considered the relevant context for the development of a CI, as stated in the Elaborated Social Identity Model (ESIM; e.g. Stott, Hoggett, and Pearson 2012). For this process, the groups' social identities and the groups' assessment of legitimacy seem to be determinant. The perception of illegitimate out-group behaviour appears to create 
common social identity among fans that accepts the use of violent behaviour as legitimate reaction or self-defence.

\section{Effects of negative perceptions of the out-group}

Critical incidents in this study seem to have aggravated, when the out-group was perceived as homogenous and deliberately provoking, behaving aggressively and seeking violence. This was considered as illegitimate behaviour and created the basis for a possible violence escalation. Conversely, a differentiated perception of the outgroup seemed to increase the likelihood of a pacification of the CI, which was also stated by Hylander and Granström (2010) and the Aggravation Mitigation Model (AMM). Actions based on differentiated perceptions are likely to support the perceived legitimacy and seem to prevent the involvement of other fans in the case of an escalation. The Elaborated Social Identity Model (ESIM; e.g. Stott, Hoggett, and Pearson 2012) and the AMM (Hylander and Granström 2010), highlight the relevance of negative out-group stereotyping. In the current study, POs in riot-gear showed stronger tendencies than police spotters in the negative stereotyping of the fans. It could be argued that "ordinary" POs mostly come into contact with football fans during riots whereas police spotters have more often positive contacts with fans, for example when escorting a fanwalk. This warrants the necessity for POs to develop a thorough understanding of the fan culture, as suggested by Havelund, Joern, and Rasmussen (2014). In the current study, several fans also disclosed their animosity towards POs and SEs, which seems to be usual for larger parts of the ultra culture (Pilz 2005). However, the animosity in this study seems to have its roots in past events involving the relevant groups. In several cases, the fans highlighted the importance of past events for their actual shared social identity as a fan group and for the interpretation of the out-groups' 
actions. Negative experiences, such as fans' arrests, appeared to shape fans' relationships with the (local) POs and SEs and seem to have an impact on future interactions between the involved groups. Actions of this out-group, e.g. police spotters, are most likely to be perceived as aggressive or offensive due to the fans' existing picture of the out-group. Accordingly, escalations between those groups seem more likely to occur in future CIs, since the perceptions of the out-group influence the first group's behaviour.

\section{Understanding the fan culture}

The fans' culture appeared to be an essential criterion for the assessment of legitimacy to the out-groups' behaviour. Violent behaviour of the fans was most likely to be considered as a legitimate reaction against infringements of their collective beliefs. Most fans reported the intentions to provide extravagant support to their team through the illegal use of pyrotechnics, while the police was bound to enforce the law. Regardless, the fans continued to use pyrotechnics, which appeared to be an essential part of their fan identity. Along with Rosander and Guvå (2012) and Stott (1996b), such contradictions between the fans' and the POs' social identities can increase the risk of escalations. Indeed, three CIs in this study appeared to have stemmed from conflicts related to pyrotechnical equipment. All fans believed that a fan's arrest due to pyrotechnics was highly illegitimate which led to an escalation. They explained that some fans will always react violently against such perceived attacks, regardless of expected chance to succeed. Indeed, these facets of fan culture can be considered as essential elements of the fans' social identity. It would be interesting to determine if a potential permission to safely use pyrotechnics, in a designated zone, would influence the occurrence of CIs. 
According to Stott et al. (2007)), the fans' perception of illegitimate out-group behaviour can cause a normative change, where the fans view violence as a legitimate reaction. Such normative change took place in Case 4, where the fans became angry after perceiving the SE's behaviour as illegitimate. However, it was not possible in the present study to recruit any fan who underwent this process and actually engaged in violent behaviour in a CI. Nonetheless, two fans mentioned this as a typical example. Several fans retained their beliefs of behaving non-violently even when subject to a high degree of provocation like in Case 4, where the SE's behaviour was assessed as very provocative. On comparing fans in this study with those in Havelund, Joern, and Rasmussen's (2012) study, the fan cultures seem to be comparable to a certain extent. They share some essential similarities, e.g. the strong solidarity, the great importance of pyrotechnics and the general distrust toward the police. Implications from this study can therefore contribute to a deeper understanding of ultra fan violence in Switzerland as well as internationally.

\section{Fan group's personal space and power issues}

An intrusion into the fan group's personal space seemed to raise tension in two cases. Congruently with the ESIM (Reicher 1996b), an intrusion was only problematic if the fans perceived the out-group's actions as provocative and illegitimate. Interestingly, the relevant CIs in our study diffused due to one group's perception of inferiority. Involved groups critically evaluated the efficacy of conflictual behaviour prior to their action. Indeed, Reicher (1996b) highlights the importance of power relations in a group context. This study presents examples of fans using a power differential in their favour against POs and vice versa. The relevant CIs' narratives suggest that the inferior group was unsupported: In Case 2, the two police spotters had no backup. In Case 5, the CI 
happened in the searching area of the stadium, whose turnstiles had been closed before the intervention of the SEs. Therefore, other fans could not join the CI. It could be argued that Case 5 might have developed differently if the power relations could have changed: The fans might not have given in if the number of fans opposing the SEs would have been on the increase. Fans could have then considered conflictual behaviour as effective, therefore leading to a possible escalation of the CI.

\section{Informative communication and the out-group's appearance}

In line with the AMM's relational condition of organising (Hylander and Granström 2010), informative intergroup communication before and during CIs was found to diffuse tension. Police officers or SEs disclosing information about their intentions and actions helped raise the fans' perceptions of legitimacy. This appeared to shape a fan identity that is defined in terms of behaving non-violently and fans were then better able to reason and calm each other down leading to the pacification of the CI. This is in line with Stott, Hoggett, and Pearson (2012), stating that the establishment of legitimacy by the police led fans to cooperate with police and to act conforming to the law. Furthermore, the AMM's third factor treatment (Hylander and Granström 2010; Rosander and Guvå 2012) is meaningful for this study considering the provocative effect of the out-group's appearance (balaclavas and riot gear). Indeed, the out-group's appearance strikes as being a crucial factor for the assessment of their behaviours and intentions. The fans in this study were concerned about their own freedom and preferred POs with riot gear in the background 


\section{The challenge of fan rivalries for police forces}

Violent conflicts between rival fan groups emerged as an essential part of radical fans culture. In this study, cases involving provocations and attacks from rival fans ended in an escalation. Provocations of rival fans without engaging in a fight afterwards were considered as illegitimate in the view of more radical fans, therefore fuelling fans' anger. This is in line with the ESIM's claim that the perception of legitimacy has to be contextualized within the relation to the collective beliefs of the relevant group (Reicher 1996b). Matches against arch-rivals seemed to particularly attract violence-seeking fans (e.g. Case 7). The fans explained these rivalries with the fan groups' history, which corresponds to the influence of the historical context on social identity (Reicher 1984). When confronted with provoking fans of an arch-rival, violence-prone fans seemed to adopt the social identity of violence-seeking fans and form a single crowd actively seeking violent confrontation with the rival fans (ESIM; Stott, Hoggett, and Pearson 2012). Indeed, the involvement of two rival fan groups and POs appeared to make a CI even more challenging. Interactions between fans and police, but also between the rival fan groups should be considered to understand the CIs' dynamics. Moreover, the police needed to build and maintain positive relationship with the fans to glean information from them about (potential) hostile clashes. Stott, Hoggett, and Pearson (2012) described this effect after implementing their policing approach based on dialogue. In the present study, clashes of rival fans led to an escalation according to the participants' narratives. Separating rival fans seems to be necessary to prevent violence escalations. Consequently, the deployment of POs with riot gear would be necessary unless infrastructural measures could be used. Overall, fans considered the police attempts to separate the fans as legitimate. 


\section{Limitations}

Fan violence was examined during 30 away matches of two clubs in the Swiss premier league. Since only fans from two clubs were interviewed, results for other football clubs may differ. For instance, the importance of pyrotechnical equipment might be less prominent for other fan groups in Switzerland or internationally. Interviews were held in Swiss German and French. Although all quotations were translated to English by a native English speaker for the purpose of this article, some meaning may have been lost or altered in the process. Furthermore, social desirability issues may have led nonviolent fans to overstate their involvement in violent actions while violent fans may have downplayed it (Pearson 2009). Nevertheless, participants did include statements about their own behaviours and values which sometimes conflicted with the law. Police officers or SEs openly discussed their feelings when facing aggressive fans.

This study is based on retrospective evaluation of specific situations. Despite the efforts made to stimulate recall of specific cases, the narratives were self-constructed representations, which can be affected by memory lapses and selection. The first author used a process of reflective subjectivity for the analysis and discussed the analysis of eight interviews with the second and third author to increase trustworthiness. Still, results might be influenced by the first author's own biases and perspective.

\section{Suggestions for future research}

Football fan research should involve football clubs from Switzerland or Europe with different fans, policies and legislation or fan cultures. For instance, it would be interesting to analyse reportedly non-violent ultra fans or to explore if other ultra fans also consider the use of pyrotechnics as important. Future research should also focus on 
violence-seeking fans (e.g. hooligans) examining their perspectives and reasons for carrying out violent actions. Longitudinal studies using multiple interview points would allow a better understanding of the fans' attitude concerning the police after implementing a more dialogue-based policing approach (see Stott, Hoggett, and Pearson 2012). In the light of the present study's findings, pre-match meetings between influential fans and police and enforce police dialogue teams to maintain successful informative communication during matches are recommended. These meetings could be held in the fans' hometown and enable a good organisation of their journey. Regarding fans perception of victimization, it would be interesting to analyse the media's influence on the fans' behaviour.

\section{Conclusions}

This study focused on the perceptions of CIs and the dynamics in CIs from the perspectives of all the prominent roles involved in such cases. Results are congruent with the ESIM (Stott et al. 2007; Stott, Hoggett, and Pearson 2012) stating that group level interactions and socio-psychological processes are essential to understand the dynamics of escalation or non-escalation of fan violence. Individuals' perceptions seem to be influenced by the assessment of the out-group's intentions and behaviours. Actions of the out-group seem to be critically evaluated and interpreted as legitimate or illegitimate. Most participants judged a case by considering possible intrusions into their personal space and the presence of provocative symbols. Past experiences with the opponent group were thought to influence the perceptions of a future situation. The involved fans, POs, and SEs exerted a mutual influence through their actions and reactions, which is in line with the ESIM (Reicher, 1996b) or the AMM (Granström et al. 2009). Stott et al. (2007) and Hylander and Granström (2010) also state that the 
perception of illegitimate out-group behaviour can lead to aggressive behaviour by the in-group. This effect was also observed in the present study, mostly on the fans' side. In this regard, it seems important to consider the local fan culture, for example the importance of using pyrotechnics for the support of the club. According to the participants, facilitating informative intergroup communication and the refraining from provoking the out-group were essential for the pacification of the CIs since they contributed to the perception of legitimacy. In contrast, the arrest of fans due to pyrotechnics was considered as highly illegitimate by the fans and was associated to an escalation of the CI by the participants. Provocations by rival fans were most likely to result in an escalation. 


\section{References}

Bundesamt für Polizei fedpol. 2014. Jahresbericht 2013: Lage, Massnahmen und Mittel. [Annual report 2013: status, measures and instruments] Available: https://www.fedpol.admin.ch/dam/data/sicherheit/jb/jabe-2013-d.pdf.

Bundesamt für Polizei fedpol. 2015. Jahresbericht 2014: Lage, Massnahmen und Mittel. [Annual report 2014: status, measures and instruments] Available: https://www.fedpol.admin.ch/dam/data/fedpol/publiservice/publikationen/bericht e/jabe/jabe-2014-d.pdf

Dal Lago, Alessandro, and Rocco De Biasi. 1994. Italian football fans: culture and organization. In Football, Violence and Social Identity, edited by R. Giulianotti, N. Bonney, and M. Hepworth, 73-89. London: Routledge.

Dovidio, J. F., Miles Hewstone, Peter Glick, and V. M. Esses. 2010. “Prejudice, stereotyping and discrimination: theoretical and empirical overview." In The SAGE handbook of Prejudice, stereotyping and discrimination, edited by J. F. Dovidio, Miles Hewstone, Peter Glick, and V. M. Esses, 3-28, London: SAGE, Drury, John and Steve Reicher. 2000. "Collective action and psychological change: The emergence of new social identities.” British Journal of Social Psychology 39: 579-604.

Granström, Kjell, Gunilla Guvå, Ingrid Hylander, and Michael Rosander. 2009. Riots and disturbances: how riots start and how order is secured. FOG-Rapport, Nr. 64. Linköping: Department of Behavioural Sciences and Learning (IBL), Linköping University.

Havelund, Jonas, Lise Joern, and Kristian Rasmussen. 2012. "Danish ultras: risk or nonrisk?" Sport and EU Review 4 (1): 5-17. 
Havelund, Jonas, Lise Joern, and Kristian Rasmussen. 2014. "A qualitative examination of police officers' perception of football supporters." Police Practice and Research: An International Journal 16 (1): 65-78.

Havelund, Jonas, Kristian Rasmussen, Clifford Stott, and Lise Joern. 2013. "Policing football - From hooliganism to intergroups dynamics." Journal of Education, Psychology and Social Sciences 1 (2): 36-41.

Hylander, Ingrid, and Kjell Granström. 2010. “Organizing for a peaceful crowd: An example of a football match." Forum Qualitative Sozialforschung/Forum: Qualitative Social Research 11, Art. 8, http://nbn-resolving.de/urn:nbn:de:0114fqs100283.

Mensch, Christian, and Andreas Maurer. 2014. Was der FCB die Polizei kostet [What the FCB costs the police]. Schweiz am Sonntag, August 23. http://www.schweizamsonntag.ch/ressort/basel/was_der_fcb_die_polizei_kostet/

Nolting, H. P. 2005. Lernfall Aggression: Wie sie entsteht-wie sie zu vermindern ist. Eine Einführung [Case study aggression: How it develops - how it can be reduced] (Vollständig überarbeitete und erweiterte Neuausgabe). Reinbek bei Hamburg: Rowohlt.

Pearson, Geoff. 2009. “The researcher as hooligan: where 'participant' observation means breaking the law." International Journal of Social Research Methodology 12 (3): $243-255$.

Pilz, G. A. 2005. „Vom Kuttenfan und Hooligan zum Ultra und Hooltra - Wandel des Zuschauerverhaltens im Profifussball.“ Deutsche Polizei 11: 6-12.

Pilz, G. A., and Franziska Wölki-Schumacher. 2010. Overview of the Ultra culture phenomenon in the council of Europe member states in 2009. (International 
Conference on Ultras: Good practises in dealing with new developments in supporters' behaviour). Council of Europe.

Projektgruppe „Rahmenkonzept Fanarbeit in der Schweiz“ [task force “conceptual framework fan coaching in Switzerland"']. 2010. Nationales Rahmenkonzept Fanarbeit in der Schweiz [national conceptual fan coaching framework in Switzerland]. Magglingen/Moosseedorf: BASPO/FaCH. Available: http://www.fanarbeit.ch/fileadmin/downloads/Konzepte/RahmenkonzeptFanarbe itdeutsch.pdf

Reicher, S. D. 1984. "The St. Pauls' riot: an explanation of the limits of crowd action in terms of a social identity model." European Journal of Social Psychology 14: 121.

Reicher, S. D. 1996a. "Social identity and social change: rethinking the context of social psychology." In Social Groups and Identities, edited by P. W. Robinson, 317336. Oxford: Butterworth-Heinemann

Reicher, S. D. 1996b. “'The Battle of Westminster': developing the social identity model of crowd behaviour in order to explain the initiation and development of collective conflict". European Journal of Social Psychology 26, 115-134.

Rosander, Michael, and Gunilla Guvå. 2012. "Keeping the peace - Police behaviour at a mass event." Journal of Investigative Psychology and Offender Profiling, 9: 5268.

Schumacher Dimech, Annemarie, Alain Brechbühl, and Roland Seiler. 2016. "Dynamics of critical incidents with potentially violent outcomes involving ultra fans: an explorative study". Sport in Society. Advance online publication, DOI: 10.1080/17430437.2015.1133597. 
Smith, J. A., Paul Flowers, and Michael Larkin. 2009. Interpretative phenomenological analysis: theory, method and research. London: Sage.

Stott, Clifford, Otto Adang, Andrew Livingstone, and Martina Schreiber. 2007.

"Variability in the collective behaviour of England fans at Euro2004:

'Hooliganism', public order policing and social change.” European Journal of Social Psychology 37: 75-100.

Stott, Clifford, Otto Adang, Andrew Livingstone, and Martina Schreiber. 2008.

"Tackling football hooliganism. A quantitative study of public order, policing and crowd psychology”. Psychology, Public Policy, and Law 14 (2): 115-141.

Stott, Clifford, James Hoggett, and Geoff Pearson. 2012. "Keeping the peace: Social identity, procedural justice and the policing of football crowds." The British Journal of Criminology 52: 381-399.

Stott, Clifford, Paul Hutchison, and John Drury. 2001. “"Hooligans' abroad? Inter-group dynamics, social identity and participation in collective 'disorder' at the 1998 World Cup Finals.” British Journal of Social Psychology 40: 359-384. 
Table 1. Sequence and description of the collected cases involving CIs without escalation.

Table 2. Sequence and description of the collected cases involving CIs with escalation.

Table 3. Differences between escalating and non-escalating CIs according the participants' narratives.

Figure 1. The course of CIs. 